\title{
Perfil histológico e imuno-histoquímico das pacientes com câncer de mama operadas no Hospital Santa Casa de Curitiba no período de 2014 e 2015
}

\author{
Histological and immunohistochemical profile of patients with breast cancer operated in \\ Santa Casa House of Curitiba in the period of 2014 and 2015 \\ Cintia Cavassim Raffo ${ }^{1}$, Danila Pinheiro Hubie ${ }^{2}$, Guilherme Leite Zanini ${ }^{3}$, Luiza Previdi Abdul-Hak ${ }^{3}$, \\ Sheldon Rodrigo Botogoski ${ }^{4}$
}

\section{Resumo}

Objetivos: Identificar tipo histológico e perfil imuno-histoquímico das pacientes operadas por câncer de mama no Hospital Santa Casa de Curitiba. Correlacionar se os tipos histológicos mais agressivos são também de pior prognóstico imuno-histoquímico. Métodos: Estudo retrospectivo, observacional e analítico com 26 pacientes, no qual foram avaliadas as variáveis: sexo, idade, histologia, painel imuno-histoquímico (luminal A, luminal B, HER2 positivo e triplo negativo). Resultados: O sexo mais prevalente foi o feminino (96,2\%), a média de idade foi 58 anos, o tipo histológico mais encontrado foi carcinoma ductal invasor $(57,7 \%)$. No painel imuno-histoquímico a prevalência foi do subtipo luminal $A(53,8 \%)$ seguidos pelos luminal $B$ (30,8\%), HER2 positivo (7,7\%) e triplo negativo (7,7\%). Na análise da relação histológica e imuno-histoquímica 57,1\% dos carcinomas ductais invasores são luminais A e o carcinoma pouco diferenciado é triplo negativo. Os mistos foram distribuídos em luminal $A(66,7 \%)$ e luminal B (33,3\%). Os

1. Médica Residente do Departamento de Ginecologia e Obstetrícia da Irmandade da Santa Casa de Misericórdia de Curitiba-Aliança Saúde - Pontifícia Universidade Católica do Paraná-PUC-PR

2. Médica do Departamento de Ginecologia e Mastologia da Irmandade da Santa Casa de Misericórdia de Curitiba - Pontifícia Universidade Católica do Paraná- PUC-PR

3. Acadêmico do Curso de Medicina da Escola de Medicina da Pontifícia Universidade Católica do Paraná - PUC-PR

4. Coordenador da Comissão de Residência Médica - Departamento de Ginecologia e Obstetrícia da Irmandade da Santa Casa de Misericórdia de Curitiba - Pontifícia Universidade Católica do Paraná-PUC-PR

Trabalho realizado: Departamento de Ginecologia e Obstetrícia da Irmandade da Santa Casa de Misericórdia de Curitiba - Pontifícia Universidade Católica do Paraná - PUC-PR

Endereço para correspondência: Sheldon Rodrigo Botogoski, Irmandade da Santa Casa de Misericórdia de Curitiba - PUC-PR. Rua André de Barros, 694 - Centro - 80010-030 - Curitiba, PR Brasil.E-mail: sheldon@centroeuropeu.com.br mucinosos dividiram-se igualmente em luminal A, luminal $B$ e triplo negativo. O lobular invasor teve distribuição igual entre luminal Ae B. Conclusões: O sexo feminino foi o mais prevalente, a média de idade apresentou um platô acima do encontrado na literatura, o tipo histológico mais incidente nas pacientes acompanhadas foi o carcinoma ductal invasor. No painel imuno-histoquímico a prevalência foi do subtipo luminal A, seguidos pelos luminal B, HER2 positivo e triplo negativo. Os tipos histológicos menos agressivos são mais comuns e também apresentam perfil imuno-histoquímico de melhor prognóstico e os mais agressivos são de pior prognóstico e menos responsivos aos tratamentos.

Descritores: Neoplasias da mama, Histologia, Imuno-histoquímica

\begin{abstract}
Objectives: Identify the histological type and immunohistochemical profile of the patients operated by breast cancer at the Hospital "Santa Casa de Curitiba" in Curitiba-PR. Correlate if the most aggressive histological types are also of worse immunohistochemical prognosis. Methods: Retrospective, observational and analytical study with 26 patients, in which the following variables were evaluated: age, sex, histology, immunohistochemical panel (luminal A, luminal $B, H E R 2$ positive and triple negative). Results: the most prevalent sex was female $(96,2)$, the mean age was 58 years, the most common histological type was invasive ductal carcinoma $(57.7 \%)$. In immunohistochemical panel the prevalence was of the luminal A subtype (53.8\%) followed by luminal B $(30.8 \%)$, HER 2 positive $(7.7 \%)$ and triple negative $(7.7 \%)$. In analysis of histological and immunohistochemical correlation, $57.1 \%$ of invasive ductal carcinomas are luminais $A$ and the poorly differentiated carcinoma is triple negative. The mixed carcinoma were distributed in luminal A (66.7\%) and luminal B $(33.3 \%)$. The mucinous ones were divided into luminal A, luminal B and triple negative equally. The invasive lobular carcinoma had equal distribution betwe-
\end{abstract}


en luminal $A$ and B. Conclusions: Female was the most prevalent sex, the average age had a plateau above the one found in the literature, the most frequent histological type in the patients followed was invasive ductal carcinoma. In immunohistochemical panel the most common was subtype luminal A, followed by Luminal B, HER2 positive and triple negative. Less aggressive histological types are more common and also have an immunohistochemical profile of better prognosis and the most aggressive ones are of worse prognostic and less responsive to treatments.

Keywords: Breast cancer, Histologty, Immunohistochemistry

\section{Introdução}

O câncer de mama é o segundo tipo de câncer mais comum entre as mulheres no mundo e no Brasil, sendo superado pelo câncer de pele não melanoma e responde por $28 \%$ dos casos novos a cada ano ${ }^{(1)}$. O câncer de mama também acomete homens, porém é raro, representando apenas $1 \%$ do total de casos da doença $a^{(1-2)}$.

Relativamente raro antes dos 35 anos, acima desta idade sua incidência cresce progressivamente, especialmente após os 50 anos. Estatísticas apontam aumento progressivo tanto nos países desenvolvidos quanto nos em desenvolvimento e indicaram que em 2016 seriam 57.960 novos casos no Brasil ${ }^{(1)}$. Felizmente com diagnóstico precoce e tratamento adequado as chances de cura estão gradativamente aumentando ${ }^{(3-4)}$.

Os principais fatores de risco para o desenvolvimento do câncer de mama segundo Silva e Riul (2012) $)^{(5)}$ relacionam-se com idade avançada, características reprodutivas, história familiar e pessoal, hábitos de vida e influências ambientais. As características reprodutivas de risco ocorrem pela doença ser estrogênio-dependente, e compreendem menarca precoce aos 11 anos ou menos, menopausa tardia aos 55 anos ou mais, primeira gestação após os 30 anos e nuliparidade. A influência da amamentação e uso de contraceptivos hormonais ainda são controversos. A história familiar e pessoal compreendem as seguintes situações: um ou mais parentes de primeiro grau com câncer de mama antes dos 50 anos, um ou mais parentes de primeiro grau com câncer de mama bilateral ou câncer ovariano em qualquer idade, parente masculino com neoplasia mamária, câncer e/ou doença mamária benigna prévios. Os hábitos de vida relacionados são a obesidade, pelo aumento do nível de estrogênio produzido no tecido adiposo, principalmente no climatério; uso regular de álcool acima de 60 gramas por dia, pois o acetaldeído, primeiro metabólito do álcool, é carcinogênico, mutagênico, estimulador da produção de estrogênio e imunodepressor. A influência do tabagismo ainda é controversa. A principal influência ambiental é a exposição prévia às radiações ionizantes, sendo diretamente proporcional à dosagem e inversamente proporcional à idade da mulher na época da exposição( ${ }^{(5)}$.

Existem vários tipos de cânceres de mama ${ }^{(6)}$, com diferentes comportamentos e evolução. A maioria dos casos tem bom prognóstico. Atualmente se investe muito na pesquisa e tratamento da neoplasia mamária, tendo como consequência o uso da imuno-histoquímica, para melhor resultado no tratamento dessas pacientes ${ }^{(7)}$. Desta forma, a avaliação dos parâmetros preditivos prognósticos no momento do diagnóstico é fundamental ${ }^{(8)}$. Dentre os fatores prognósticos, os mais significativos em relação à gravidade são o tamanho do tumor e a condição dos linfonodos comprometidos. Em seguida, considera-se a graduação e subtipo histológico, somados à avaliação da presença de biomarcadores ${ }^{(9)}$.

Em 2012 foi publicada a quarta edição da classificação de tumores de mama da Organização Mundial de Saúde (OMS) ${ }^{(10)}$. Utilizando critérios morfológicos, são reconhecidos o carcinoma mamário invasivo de tipo não especial e outros 20 tipos histológicos ${ }^{(11)}$. Usualmente identificado na prática médica como carcinoma ductal invasivo (CDI), o carcinoma mamário invasivo de tipo não especial representa $70 \%$ dos casos e não preenche critérios para classificação em nenhum subgrupo, sendo um tumor heterogêneo ${ }^{(12)}$. Nos tipos especiais, o carcinoma lobular invasivo (CLI) é o mais prevalente respondendo por $10-15 \%$, são mais homogêneos, com critérios diagnósticos mais rígidos ${ }^{(13)}$. É importante frisar que não é incomum uma mesma neoplasia apresentar áreas com diferentes morfologias, sendo considerada de padrão misto ${ }^{(12)}$.

A expressão dos receptores de estrógeno (RE), de progesterona (RP) e a superexpressão ou amplificação do receptor de fator de crescimento epidérmico humano 2 (HER2) foram identificados como importantes fatores preditivos entre as pacientes com câncer de mama $^{(14-17)}$.

Cerca de dois terços dos cânceres de mama são positivos para receptor hormonal $(\mathrm{RH})$, sendo que a positividade para os RE varia de 60 a $81 \%$, enquanto que para os RP de 44 a $61 \%{ }^{(18)}$.

O estudo imuno-histoquímico tem sido utilizado em diferentes situações na patologia mamária como: avaliação de fatores preditivos e prognósticos do câncer de mama, pesquisa de células epiteliais metastáticas em linfonodo sentinela, diagnóstico diferencial de lesões mamárias e determinação de possível origem de neoplasias metastáticas ${ }^{(15)}$.

Existe grande heterogeneidade genética nos cânceres de mama e pela tecnologia microarray, que 
permite análise de todo o DNA genômico, os tumores de mama foram classificados em sete subtipos intrínsecos: ${ }^{(19)}$

1. Luminal A: origina-se em células epiteliais ricas em RE e RP, com ausência de HER2 e baixa proliferação de KI-67 (< 14\%). É muito sensível a hormonioterapia. É o mais comum $40 \%$ e o que tem melhor prognóstico.

2. Luminal B: tem a mesma origem e exibe RE em alta porcentagem, os RP costumam ter baixa frequência e o Ki-67 é elevado (>14\%). O HER2 pode estar expresso.

3. Superexpressor de HER2: corresponde a $15 \%$ dos casos. Geralmente RE e RP negativos. Sua evolução natural é complicada, panorama que foi mudado pela introdução de terapia alvo com herceptina.

4. Basalóide: cerca de $20 \%$. Sua grande maioria é triplo negativo em imuno-histoquímica (RE, RP e HER2). Apresenta algumas características de células mioepiteliais (CK5 e CK6). É um tumor de mau prognóstico, porém sensível à quimioterapia. Nem todo tumor basalóide é triplo negativo e vice e versa, contudo no dia a dia são termos empregados analogamente.

5. Normal-like : é o tumor com genes comuns ao epitélio normal.

6. Claudina baixa: são triplos negativos, com baixa expressão de claudinas que são proteínas de adesão. São ricos em marcadores de células-tronco e de transição epitélio-mesênquima. Agressividade elevada.

7. Molecular apócrino: também são triplos negativos, porém ricos em receptores androgênicos. Seu prognóstico parece ser o melhor entre os triplos negativos.

Existe uma associação significativa entre os $\mathrm{RH}$ e a histologia dos carcinomas de mama. Geralmente, os carcinomas medulares são negativos para RH. Nos CDI existe uma relação inversa entre o grau histológico e nuclear e a positividade para os $\mathrm{RE}$ e os $\mathrm{RP}^{(20)}$.

Os RE e os RP estão altamente associados com a idade da paciente no diagnóstico, sendo significativamente mais positivos com o avanço da idade ${ }^{(21)}$. Os RH não estão relacionados com a história familiar, paridade ou idade da menarca. Todos os tumores positivos para RP são também positivos para $\mathrm{RE}^{(18)}$.

Em um estudo 119 pacientes portadores de câncer de mama tiveram seus perfis imuno-histoquímicos para RE, RP e HER2 avaliados. Nesse estudo dentre os carcinomas ductais invasores a maior prevalência foi do subtipo luminal A, seguido por subtipos basal, luminal B e superexpressor de HER2. Dos lobulares invasores o mais prevalente é o luminal A, seguido pelo luminal B e basal na mesma proporção. Dos medulares todos eram do subtipo basal. Analisando perfil imuno-histoquímico, a maioria de todos os subtipos (luminal A, luminal B, basal e HER2) foram carcinomas ductais invasores ${ }^{(22)}$.

Os fatores prognósticos contribuem para a evolução da doença e orientam a terapia complementar. Dentre eles, os mais significativos em relação a gravidade são o número de linfonodos comprometidos e o tamanho tumoral. Adicionalmente, consideram-se subtipos, graus histológico e nuclear e invasão vascular-linfática. Por imuno-histoquímica, tumores que apresentam receptores hormonais negativos e/ou HER2 positivo tendem a evoluir desfavoravelmente, assim como KI-67 elevado.

Em vista da importância do câncer de mama e de descobrir os fatores prognósticos para melhor escolha do tratamento adjuvante este trabalho vem com intuito de analisar o perfil das pacientes que são atendidas no serviço de Ginecologia e Mastologia do Hospital Santa Casa de Curitiba.

Deste modo, pretendeu-se identificar o tipo histológico, o perfil imuno-histoquímico e analisar se há relação entre os tipos histológicos mais agressivos e os perfis imuno-histoquímicos de pior prognóstico e que exibem pior resposta ao tratamento adjuvante das pacientes operadas por câncer de mama no Hospital Santa Casa de Curitiba, no período de junho de 2014 a julho de 2015.

\section{Materiais e Métodos}

Estudo retrospectivo, observacional e analítico, através de análise de prontuários de pacientes operados por câncer de mama no Serviço de Ginecologia e Mastologia do Hospital Santa Casa de Curitiba.

Os prontuários analisados foram referentes ao período de junho de 2014 a julho de 2015. Para realizar a coleta dos dados de forma sistemática foi necessário revisão manual de cada prontuário.

Foram incluídos neste estudo pacientes com diagnóstico de câncer de mama e operados no serviço. No presente estudo excluíram-se os pacientes que não apresentaram laudo imuno-histoquímico completo. Este trabalho constitui um estudo retrospectivo, observacional e analítico que avaliou um total de 26 pacientes sob as seguintes variáveis: sexo, idade, tipo histológico de câncer de mama, painel imuno-histoquímico (luminal A, luminal B, HER2 e triplo negativo).

A análise estatística dos dados obtidos, assim como o armazenamento das informações coletadas manualmente, foi realizada através do Microsoft Excel 2007 e Microsoft Word 2007.

O presente estudo foi submetido e aprovado pelo comitê de ética em pesquisa da Pontifícia Universidade Católica do Paraná- PUCPR sob CAAE: 66092416.7.0000.0020. 


\section{Resultados}

Neste estudo foram analisados 40 prontuários. Desses, foram excluídos 13 prontuários cujos laudos imuno-histoquímicos encontravam-se incompletos e 1 paciente que o resultado histológico não foi de câncer de mama. Todos os 26 pacientes foram operados no Serviço de Ginecologia e Mastologia do Hospital Santa Casa de Curitiba.

No presente estudo tivemos apenas um homem dentre os pacientes com câncer de mama operados, sendo que $96,2 \%$ da amostra foi representada por mulheres.

A média de idade dos pacientes estudados foi de 58 anos, variando entre 32 e 82 anos. Destes $42,3 \%$ ( $\mathrm{n}=11$ ) estão na faixa etária entre 45-60 anos (Quadro 1).

\begin{tabular}{|c|c|}
\hline \multicolumn{2}{|c|}{ Quadro 1} \\
\hline \multicolumn{2}{|c|}{ Faixa etária dos pacientes estudados no serviço de } \\
Ginecologia e Mastologia do Hospital Santa Casa de \\
Curitiba. \\
\hline 30-40 anos & $11,5 \%(\mathrm{n}=3)$ \\
$41-50$ anos & $11,5 \%(\mathrm{n}=3)$ \\
$51-60$ anos & $30,8 \%(\mathrm{n}=8)$ \\
$61-70$ anos & $38,5 \%(\mathrm{n}=10)$ \\
$71-80$ anos & $4 \%(\mathrm{n}=1)$ \\
$81-90$ anos & $4 \%(\mathrm{n}=1)$ \\
\hline
\end{tabular}

Analisando o sexo dos pacientes o único luminal B que apresentou HER2 positivo foi o do sexo masculino. As demais apresentações foram do sexo feminino.

$\mathrm{Na}$ análise quanto ao tipo histológico do câncer de mama o subtipo mais prevalente foi o carcinoma ductal invasor, 57,7\% ( $n=15)$, conforme apresentado no Gráfico 1. O segundo mais prevalente foi o misto $19,2 \%$ $(\mathrm{n}=5)$ com componente ductal e lobular. O mucinoso representou $11,5 \%(n=3)$ e carcinoma lobular invasor $7,7 \%(n=2)$. O menos representativo foi o carcinoma pouco diferenciado com $4 \%(n=1)$.

De acordo com a análise do perfil imuno-histoquímico dos paciente avaliados, $53,8 \%(\mathrm{n}=14)$ foram luminal A, 30,8\% $(n=8)$ luminal $B$ (dos quais um apresentou expressão do HER2), 7,7\% ( $\mathrm{n}=2)$ HER2 positivo e 7,7\% (n=2) triplo negativo. Conforme o gráfico 2.

Associando os tipos histológicos com os perfis imuno-histoquímicos neste estudo, notou-se que dos carcinomas ductais invasores $57,1 \%$ são luminal A, $28,6 \%$ são luminal B e 14,3\% HER2. Os carcinomas mistos foram distribuídos em luminal A $(66,7 \%)$ e luminal B (33,3\%). Os mucinosos dividiram-se igualmente em luminal A, luminal B e triplo negativo. $\mathrm{O}$ lobular invasor teve distribuição igual entre luminal A e B. O carcinoma pouco diferenciado apresentou-se triplo negativo, conforme Quadro 2.

\section{Discussão}

O câncer de mama representa um grande problema de saúde pública e analisando os resultados do nosso estudo podemos perceber que em relação ao sexo o prognóstico do paciente masculino foi pior em relação à maioria das mulheres. Porém, o número de pacientes do sexo masculino não foi suficiente para ter significância estatística. No entanto, não podemos deixar de perceber que o câncer de mama afeta significativamente mais mulheres do que homens, como demonstrado nesse estudo. Isso corrobora o fato de os principais fatores de risco apresentados na literatura serem relacionados às mulheres ${ }^{(23-25)}$.

Em relação a idade, que é um importante fator de risco segundo estudo de Brandt et al (2015) ${ }^{(26)}$; os dados

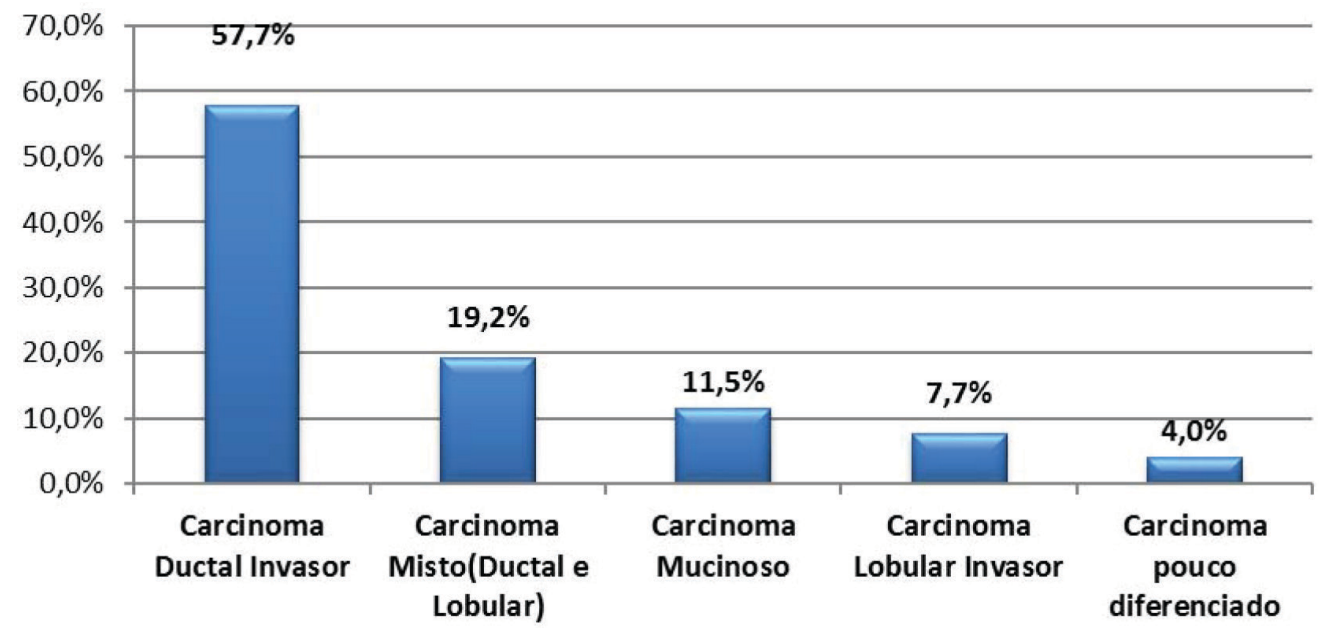

Gráfico 1 - Tipos histológicos do câncer de mama dos 26 pacientes estudados no serviço de Ginecologia e Mastologia do Hospital Santa Casa de Curitiba nos anos de 2014 e 2015. 


\section{Perfil Imuno-Histoquímico}

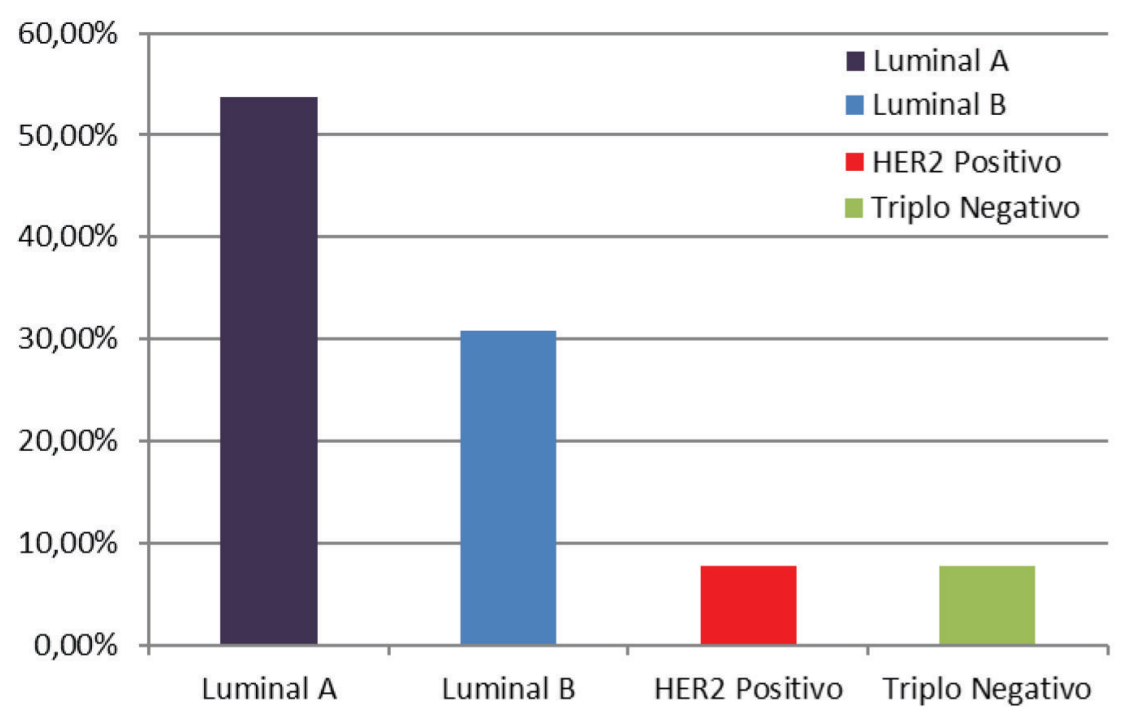

Gráfico 2 - Análise do perfil imuno-histoquímico dos 26 pacientes estudados no serviço de Ginecologia e Mastologia do Hospital Santa Casa de Curitiba nos anos de 2014 e 2015.

\section{Quadro 2}

Correlação percentual entre tipo histológico e perfil imuno-histoquímico dos 26 pacientes estudados no serviço de Ginecologia e Mastologia do Hospital Santa Casa de Curitiba nos anos de 2014 e 2015.

\begin{tabular}{|l|c|c|c|c|}
\multicolumn{1}{r|}{$\quad$ Perfil imuno-histoquímico } & Luminal A & Luminal B & TER2 & \\
\hline Carcinoma Distológico & & & & \\
Misto & $57,1 \%(8)$ & $28,6 \%(4)$ & $14,3 \%(2)$ & $0,0 \%$ \\
Mucinoso & $66,67 \%(4)$ & $33,33 \%(2)$ & $0,0 \%$ & $0,0 \%$ \\
Carcinoma Invasor & $33,33 \%(1)$ & $33,33 \%(1)$ & $0.0 \%$ & $33,33 \%(1)$ \\
Pouco Diferenciado & $50 \%(1)$ & $50 \%(1)$ & $0,0 \%$ & $0,0 \%$ \\
\hline
\end{tabular}

obtidos da literatura apresentam um platô entre 45 e 50 $\operatorname{anos}^{(27-28)}$, porém no nosso estudo essa faixa de idade foi um pouco superior, sendo a média de 58 anos. Não mudando o fato de ambos mostrarem poucas pacientes com idade inferior a 30 anos. O estudo de Sarturi et al $(2012)^{(22)}$, mostrou que o subtipo basalóide (triplo negativo) esteve presente em pacientes com idade inferior a 39 anos, porém no nosso estudo a paciente que apresentou esse subtipo foi a paciente mais idosa.

Conforme já apresentado em trabalho de Geyer e Nigro ${ }^{(12)}$, em nosso estudo surgiram dados semelhantes em relação a frequência dos tipos histológicos, sendo que o carcinoma ductal invasor foi o mais comum, seguido pelo misto e trazendo o carcinoma lobular invasor como menos frequente, ordem também encontrada na literatura(29-32).

Analisando o perfil imuno-histoquímico dos casos apresentados, o mais prevalente foi o Luminal A conforme dados encontrados na literatura ${ }^{(22,30,33)}$. Porém o triplo negativo se apresentou mais prevalente do que o Luminal $B^{(30,33)}$, divergindo do nosso estudo onde o Luminal B aparece mais prevalente que o triplo negativo corroborando com o trabalho de Yábar et al (2017) ${ }^{(34)}$.

$\mathrm{Na}$ associação dos tipos histológicos e perfis imuno-histoquímicos, é apresentado na literatura que a maioria dos carcinomas ductais invasores foram também luminais $\mathrm{A}^{(22,32,35)}$, como em nosso estudo, o que nos mostra um melhor prognóstico tumoral e mais fácil tratamento adjuvante com hormonioterapia. Dos lobulares invasores a nossa prevalência foi igual entre luminal A e luminal B, reafirmado por Denis et al (2016) ${ }^{(32)}$ já na literatura o luminal A foi o mais frequente ${ }^{(35-36)}$. Observamos que o de pior prognóstico foi também o de tratamento mais difícil, pois o carcinoma pouco diferenciado apresentou-se como o subtipo triplo negativo ${ }^{(37-39)}$, que não é responsivo a hormonioterapia nem ao anticorpo monoclonal e de resposta variável aos esquemas quimioterápicos. 


\section{Conclusão}

No presente estudo encontramos que o carcinoma ductal invasor é o tipo histológico mais prevalente e o subtipo luminal A é o mais encontrado nos pacientes. A maioria das pacientes com câncer de mama possuem o tipo de melhor prognóstico sendo responsivo a tratamento adjuvante hormonioterápico. Correlacionando os tipos histológicos com os perfis imuno-histoquímicos obtivemos que os de menor agressividade são também de melhor prognóstico, como o carcinoma ductal invasor que é na sua maioria luminal A. Já os tumores mais agressivos, como o pouco diferenciado tem pior prognóstico imuno-histoquímico por ser triplo negativo e não responsivo as medicações como a hormonioterapia e os anticorpos monoclonais.

\section{Referências}

1. Instituto Nacional do Câncer (INCA). José de Alencar Gomes da Silva. [online]. Mama. Disponível em http://www2.inca. gov.br/wps/wcm/connect/tiposdecancer/site/home/mama [29 maio 2017].

2. Silvestri V, Barrowdale D, Mulligan AM, Neuhausen SL, Fox S, Karlan BY, et al. Male breast cancer in BRCA1 and BRCA2 mutation carriers: pathology data from the Consortium of Investigators of Modifiers of BRCA1/2. Breast Cancer Res. 2016; 18(1):15.

3. Bodai BI, Tuso P. Breast cancer survivorship: a comprehensive review of long-term medical issues and lifestyle recommendations. Perm J. 2015:19(2):48-79.

4. Connors SK, Goodman MS, Noel L, Chavakula NN, Butler D, Kenkel S, et al. Breast cancer treatment among African American women in North St. Louis, Missouri. J Urban Health. 2015; 92(1):67-82.

5. Silva PA, Riul SS. Câncer de mama: fatores de risco e detecção precoce. Rev Bras Enferm. 2011;64(6):1016-21.

6. Noone AM, Cronin KA, Altekruse SF, Howlader N, Lewis DR, Petkov VI, et al. Cancer incidence and survival trends by subtype using data from the surveillance epidemiology and end results program, 1992-2013. Cancer Epidemiol Biomarkers Prev. 2016; 26(4):32-41.

7. Instituto Nacional do Câncer (INCA). Controle do câncer de mama. Documento de consenso. Rio de Janeiro: INCA; 2004. 36p.

8. Abreu E, Koifman S. Fatores prognósticos no câncer de mama feminina. Rev Bras Cancerol. 2002;48(1):113-31.

9. Cappellani A, Di Vita M, Zanghi A, Cavallaro A, Piccolo G, Majorana M, et al. Prognostic factors in elderly patients with breast cancer. BMC Surg.2013;13(suppl2): S2.

10. Lakhani SR, Ellis IO, Schnitt SJ, Tan PH, Van de Vijver MJ, editors.WHO Classification of tumours of the breast. $4^{\text {th }}$ ed. Lyon: IARC; 2012. 240p.

11. Gobbi H. Classificação dos tumores da mama: atualização baseada na nova classificação da Organização Mundial da Saúde de 2012. Bras Patol Med Lab. 2012; 48(6):463-74.

12. Geyer FC, Nigro MV. Tipos histológicos especiais de câncer de mama. Onco \&. 2013; 3(15):32-6.

13. Sawyer E, Roylance R, Petridis C, Brook MN, Nowinski S, Papouli E, et al. Genetic predisposition to in situ and invasive lobular carcinoma of the breast. Plos Genet. 2014; 10(4):e1004285.

14. Cintra JRD, Teixeira MTB, Diniz RW, Gonçalves Junior H,
Florentino TM, Freitas GF, et al. Perfil imuno-histoquímico e variáveis clinicopatológicas no câncer de mama. Rev Assoc Med Bras. 2012;58(2):178-87.

15. Salles MA, Cúrcio VS, Perez AA, Gomes DS, Gobbi H. Contribuição da imuno-histoquímica na avaliação de fatores prognósticos e preditivos do câncer de mama e no diagnóstico de lesões mamárias. J Bras Patol Med Lab. 2009; 45(3):213-22.

16. Zhou Z, Qiao JX, Shetty A, Wu G, Huang Y, Davidson NE, et al. Retracted article: regulation of estrogen receptor signaling in breast carcinogenesis and breast cancer therapy. Cell Mol Life Sci. 2014; 71(8):1549.

17. Sirkisoon SR, Carpenter RL, Rimkus T, Miller L, MethenyBarlow L, Lo HW. EGFR and HER2 signaling in breast cancer brain metastasis. Front Biosci (Elite Ed). 2016; 8:245-63.

18. Einsenberg ALA, Koifman S. Câncer de mama: marcadores tumorais (revisão da literatura). Rev Bras Cancerol. 2001; 47(4):377-88.

19. Barros ACSD. Câncer de mama. In: Urbanetz AA. Ginecologia e obstetrícia FEBRASGO para o médico residente. Barueri (SP): Manole; 2016. p.741-65.

20. Hirata BKB, Oda JMM, Guembaroviski RL, Ariza CB, Oliveira CEC, Watanabe MAE. Molecular markers for breast cancer: prediction on tumor behavior. Dis Markers. 2014; 2014:513158.

21. Lateef F, Jamal S, Nasir S, Jamil Z. Invasive ductal carcinoma: correlation of immunophenotypic features with age. J Coll Physicians Surg Pak. 2017; 27(1):18-22.

22. Sarturi PR, Cunha Júnior AD, Morais CF. Perfil imunohistoquímico do câncer de mama de pacientes atendidas no Hospital do Câncer de Cascavel - Paraná. Rev Bras Oncol Clín. 2012; 8(29):121-24.

23. Rahib L, Smith BD, Aizenberg R, Rosenzweig AB, Fleshman JM, Matrisian LM. Projecting cancer incidence and deaths to 2030: the unexpected burden of thyroid, liver and pancreas cancers in the United States. Cancer Res. 2014; 74(11):2913-21.

24. Colditz GA, Bohlke K. Priorities for the primary prevention of breast cancer. CA Cancer J Clin. 2014; 64(3):186-94.

25. Howell A, Anderson AS, Clarke RB, Duffy SW, Evans DG, Garcia-Closas M, et al. Risk determination and prevention of breast cancer. Breast Cancer Res.2014; 16:446.

26. Brandt J, Garne JP, Tengrup I, Manjer J. Age at diagnosis in relation to survival following breast cancer: a cohort study. World J Surg Oncol. 2015; 13:33.

27. Warner ET, Colditz GA, Palmer JR, Partridge AH, Rosner BA, Tamimi RM. Reproductive factors and risk of premenopausal breast cancer by age at diagnosis: are there differences before and after age 40? Breast Cancer Res Treat. 2013;142(1):165-75.

28. Kuijer A, King TA. Age, molecular subtypes and local therapy decision-making. Breast. 2017; 34 (Suppl 1):S70-S77.

29. Abdollahi A, Zadeh HS, Akbari M, Tahmasbi S, Talei A, Hassanzadeh J. Investigation of prognostic factors and survival without recurrence in patients with breast cancer. Adv Biomed Res. 2017; 6:42.

30. Sahraoui G, Khanchel F, Chelbi E. Profil anatomopathologique du cancer du sein dans le cap bon Tunisien. Pan Afr Med J. 2017;26:11.

31. Xie Y, Lv X, Luo C, Hu K, Gou Q, Xie K, Zheng H. Surgery of the primary tumor improves survival in women with stage IV breast cancer in southwest China: a retrospective analysis. Medicine(Baltimore). 2017; 96(22):e7048.

32. Denis M, Gregory A, Bayat M, Fazzio RT, Whaley DH, Ghosh $\mathrm{K}$, et al. Correlating tumor stiffness with immunohistochemical subtypes of breast cancers: prognostic value of comb-push ultrasound shear elastography for differentiating luminal subtypes. Plos One. 2016; 11(10):e0165003.

33. Pérez-Rodríguez G. Prevalence of breast cancer sub-types by immunohistochemistry in patients in the regional general 
hospital 72, Instituto Mexicano del Seguro Social. Cir Cir. 2015; 83(3):193-8.

34. Yábar A, Meléndez R, Muñoz S, Deneo H, Freire J, Dominguez $\mathrm{V}$, et al. Effect of Ki-67 assessment in the distribution of breast cancer subtypes: evaluation in a cohort of Latin American patients. Mol Clin Oncol. 2017; 6(4):503-9.

35. Soliman NA, Yussif SM. Ki-67 as a prognostic marker according to breast cancer molecular subtype. Cancer Biol Med. 2016; 13(4):496-504.

36. Alnegheimish NA, Alshatwi RA, Alhefdhi RM, Arafah MM, Alrikabi AC, Husain S. Molecular subtypes of breast carcinoma in Saudi Arabia. Saudi Med J. 2016; 37(5):506-12.

37. Choi YL, Oh E, Park S, Kim Y, Park YH, Song K et al. Triplenegative, basal-like, and quintuple-negative breast cancers: better prediction model for survival. BMC Cancer. 2010; 10:507.
38. Lin NU, Vanderplas A, Hughes ME, Theriault RL, Edge SB, Wong YN, et al. Clinicopathologic features, patterns of recurrence, and survival among women with triple-negative breast cancer in the national comprehensive cancer network. Cancer. 2012; 118(22):5463-72.

39. Palma G, Frasci G, Chirico A, Esposito E, Siani C, Saturnino C, et al. Triple negative breast cancer: looking for the missing link between biology and treatments. Oncotarget. 2015; 6(29):2656074 .

Trabalho recebido: 05/07/2017

Trabalho aprovado: $24 / 11 / 2017$ 